\title{
Optical nonresonance and two-photon resonance breathers in anisotropic media
}

\author{
G. T. Adamashvili \\ D. J. Kaup \\ University of Central Florida
}

Find similar works at: https://stars.library.ucf.edu/facultybib2000 University of Central Florida Libraries http://library.ucf.edu

This Article is brought to you for free and open access by the Faculty Bibliography at STARS. It has been accepted for inclusion in Faculty Bibliography 2000s by an authorized administrator of STARS. For more information, please contactSTARS@ucf.edu.

\section{Recommended Citation}

Adamashvili, G. T. and Kaup, D. J., "Optical nonresonance and two-photon resonance breathers in anisotropic media" (2004). Faculty Bibliography 2000s. 2581.

https://stars.library.ucf.edu/facultybib2000/2581

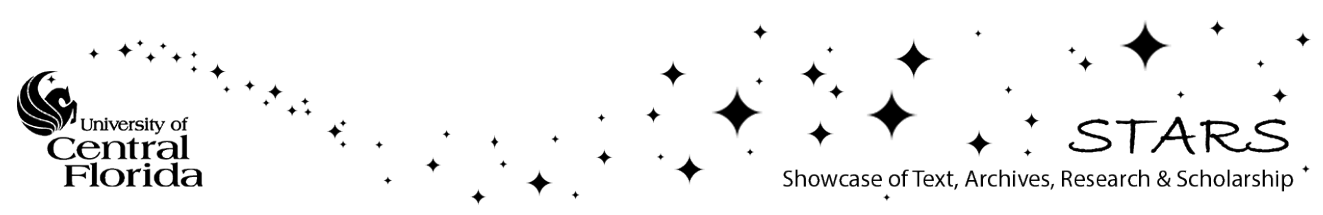




\title{
Optical nonresonance and two-photon resonance breathers in anisotropic media
}

\author{
G. T. Adamashvili* \\ Max-Planck-Institut für Physik Komplexer Systeme, Nöthnitzer Strasse 38, D-01187 Dresden, Germany \\ D. J. Kaup ${ }^{\dagger}$ \\ Department of Mathematics \& Institute for Simulation and Training, University of Central Florida, Orlando, Florida 32816-1364, USA
}

(Received 9 June 2004; published 16 December 2004)

\begin{abstract}
Anisotropic optical crystals with two-photon resonance impurity atoms are shown to have three different mechanisms of the formation of breathers depending on the direction of the wave propagation and on the symmetry of the medium. Explicit analytic expressions for the parameters of nonresonance and resonance two-photon breathers of extraordinary waves are obtained. It is shown that, unlike one-photon breathers, for two-photon breathers in the media with quadratic susceptibilities and the crystal classes $3,3 \mathrm{~m}, 4,4 \mathrm{~mm}, 6$, $6 \mathrm{~mm}$, and Kerr media two different structures of the breathers zones are realized.
\end{abstract}

DOI: 10.1103/PhysRevE.70.066616

PACS number(s): 42.65.Tg, 42.50.Md

\section{INTRODUCTION}

The existence of breathers is one of the most interesting and important manifestations of nonlinearity in optical systems [1-3]. The determination of the mechanisms causing the formation of the optical breathers and the investigation of their properties in different nonlinear media are among the principal problems of the physics of nonlinear waves.

The basic sources of the optical nonlinearity in dielectrics and semiconductors may be the following. (1) Nonresonant nonlinearity. Media possess nonlinear susceptibilities of second (quadratic) and third (cubic) orders [4,5]. (2) Resonant nonlinearity. A medium which contains optically active impurities whose excitation frequency is in resonance with the frequency of a nonlinear optical wave. In such a case we can observe the existence of one-photon and multiphoton resonance processes, which form the basis of nonlinear resonance spectroscopy [6].

Depending on the nature of the nonlinearity, the nonresonance or resonance mechanism of the formation of breathers (MFB) is realized. In the case of nonresonant nonlinearity, which is expressed by means of the quadratic $\left(d_{i j k}\right)$ or cubic $\left(\rho_{i j k l}\right)$ susceptibilities, its competition with the dispersion leads to the formation of nonresonance optical breathers [1,7-9].

The resonant optical nonlinear wave can be formed with the help of the resonance (McCall-Hahn) mechanism of the formation of nonlinear waves, i.e., from a nonlinear coherent interaction of an optical pulse with resonant impurity atoms in solids, when the conditions of the self-induced transparency: $\omega T \gg 1$ and $T \ll T_{1,2}$ are fulfilled, where $T$ and $\omega$ are the width and frequency of the pulse, $T_{1}$ and $T_{2}$ are the longitudinal and transverse relaxation times of the impurity atoms $[10,11]$. When the area (energy, in the case of the two-photon

\footnotetext{
*Permanent address: Tbilisi State University, Chavchavadze Ave. 1, Tbilisi, Georgia, 0179.

Email address: gadama@mpipks-dresden.mpg.de

†Email address: kaup@ucf.edu
}

self-induced transparency) of the pulse $\Theta>\pi$, the solitons are generated, but for $\Theta \ll 1$ resonance optical breathers are formed [1-3].

In experiments of McCall and Hahn [10] in a crystal of ruby $\mathrm{Al}_{2} \mathrm{O}_{3}: \mathrm{Cr}^{3+}$ the excitation of resonance soliton was reached when the pulse intensity exceeds some critical value about $100 \mathrm{~W} / \mathrm{cm}^{2}$. The necessary intensity for exciting resonance optical breathers of small energy is significantly smaller than the intensity necessary for exciting a resonance soliton $(2 \pi$ pulse). Therefore the breathers can be excited easier. The resonance optical waves of small energy are particularly interesting also because they can take part in a wide variety of nonlinear optical phenomena, for instance, in the processes of the formation of optical double breathers [12]. Resonance breathers of some equations of nonlinear optics are also highly stable. The breather can be also considered as a "zero-area pulse" which is experimentally studied in the work [13] (see also [14]).

Depending on nonlinearity of the medium various MFB are realized. When the coefficient of photon-atom connection $K \ll L$ nonresonance $[9,15]$ ( $K \gg L$ resonance $[2,3])$ MFB is realized, (where $L=d_{i j k}$ in noncentrosymmetric media and $L=\rho_{i j k l}$ in Kerr media). When $K=L$ the "blended" MFB can take place, when resonance and nonresonance mechanisms are acting effectively simultaneously. The "blended" mechanisms of the formation of the nonlinear optical and acoustical waves have been considered by many authors [15-19].

Because numerical values of the quantities $d_{i j k}, \rho_{i j k l}$ and $K$ can vary very strongly in different media, different solids will realize different mechanisms of the formation of optical breathers [15]. But even more interesting for the study and comparison of different mechanisms is the investigation of these processes in one and the same crystal. Such a possibility is given if we consider anisotropic uniaxial crystals and investigate processes of the formation of optical breathers for optical extraordinary waves (Fig.1). It is well known that the properties of extraordinary waves depend on the direction of their propagation and therefore for different directions of the propagation of the waves different relations between the quantities $d_{i j k}, \rho_{i j k l}$ and $K$ are realized. Hence if we change the direction of the propagation of nonlinear waves, different 


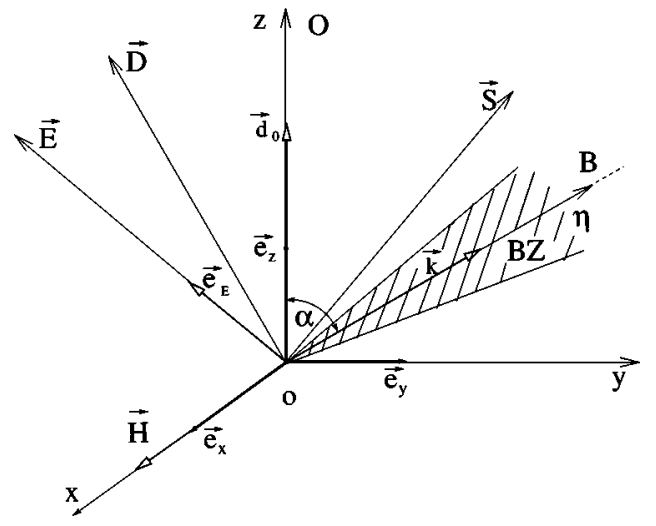

FIG. 1. The direction of the propagation of the extraordinary wave along axis $\eta$. The vectors $\vec{E}, \vec{D}, \vec{S}$, and $\vec{k}$ lie in the $y z$ plane. The principal optical axis $O$ of the uniaxial crystal and the vector of electrical dipole moment of the impurity atoms $\vec{d}_{0}$ point along axis $z$. Direction of the axis $\eta$ coincide with one of the chosen directions $B$ shown as dashed lines. The BZ correspond to the hatched region in which one of the MFB is realized.

mechanisms of the formation of optical breathers will be realized. Consequently, in uniaxial media there exist certain propagation directions $B$ (and zones around them) along which one of the above mentioned MFB will be realized.

The investigation of the breather formation processes and specific peculiarities of the propagation of nonlinear waves in anisotropic media is also of interest because many laser crystals are anisotropic [4,5], and isotropic solids can become optically anisotropic ones in the presence of a constant electric field or under the influence of a deformation [20]. It is very important also that anisotropic uniaxial crystals in many modern optical devices are used. Consequently this considered problem has a rather general character and not only has theoretical interest but it is very important for applications too.

All these problems under the condition of the one-photon excitation of the impurities (OPEI) in uniaxial nonlinear resonance media are investigated in details in Ref. [15]. Under the condition of the two-photon excitations of the uniaxial nonlinear resonance media, physical situations will be different and special consideration will be necessary.

The main goal of this work is as follows. The investigation of the structure of breathers zones (SBZ) and the conditions of realization of the resonance, nonresonance and "blended" MFB in different anisotropic media under the condition of the two-photon excitation of the impurities (TPEI) and the determination of the explicit analytic expressions for the parameters of the two-photon breathers for the extraordinary waves.

\section{BASIC EQUATION}

We consider the mechanisms of the formation of optical breathers in the anisotropic, (quadratic or cubic) nonlinear and second order dispersive media under the condition of two-photon excitation of containing impurity atoms in the case when an optical pulse with width $T \ll T_{1,2}$ and frequency $\omega \gg T^{-1}$ propagating in the positive direction along the $\eta$ axis make angle $\alpha$ with the $z$ axis. We shall consider the optically uniaxial media-trigonal, tetragonal, and hexagonal crystals with components of the permittivity tensor $\varepsilon_{x x}=\varepsilon_{y y} \neq \varepsilon_{z z}$. In these crystals one of the principal axes of the permittivity tensor $\varepsilon_{i j}$ coincides with the axis of the symmetry of third, fourth and sixth order, respectively. This axis is called optical axis of the uniaxial crystal and we assume that this axis $O$ is points along $z$ axis (Fig. 1). Corresponding principal value of the tensor $\varepsilon_{i j}$ we determine as $\varepsilon_{z z}=\varepsilon_{\|}$. Direction of the two other principal axes (in the plane perpendicular with $z$ axis) are arbitrary and we determined as $\varepsilon_{x x}=\varepsilon_{y y}=\varepsilon_{\perp}$. Not concreting the physical nature of dispersive process, we describe the dependence of permittivity tensor $\varepsilon_{i j}$ on two variableswave vector $\vec{k}$ and frequency $\omega$ of wave (spatially and/or temporally dispersion) [20].

In uniaxial media, the electric displacement vector $\vec{D}$ and the vector of the strength of the electric field $\vec{E}$ of the pulse are parallel only if $\vec{k}$ points in the direction of one of the principal optical axis, but not in general. There are two systems of orthogonal vector triplets: $(\vec{D}, \vec{H}, \vec{k})$ and $(\vec{E}, \vec{H}, \vec{S})$ and without any loss of generality we assume that $\vec{E}, \vec{D}, \vec{k}$, and the Poynting vector $\vec{S}=(c / 4 \pi)[\vec{E}, \vec{H}]$ lie in the single plane $y z$ perpendicular of the strength of the magnetic field $\vec{H}$, where $c$ is the light velocity in vacuum (Fig. 1). Then $\vec{k} \cdot \vec{r}$ $=k \eta$, where $\eta=z \cos \alpha+y \sin \alpha$.

The wave equation for the strength of the electrical field $\vec{E}(\eta, t)$ of the optical pulse in uniaxial media has the following form:

$$
\frac{\partial^{2} \vec{E}}{\partial t^{2}}-c^{2} \frac{\partial^{2} \vec{E}}{\partial \eta^{2}}=-4 \pi\left(\frac{\partial^{2} \vec{P}}{\partial t^{2}}-c^{2} \operatorname{grad} \operatorname{div} \vec{P}\right),
$$

where the polarization of the medium

$$
\vec{P}=\int \hat{\chi}^{(1)}\left(x_{1}, t_{1}\right) \vec{E}\left(\eta-x_{1}, t-t_{1}\right) d t_{1} d x_{1}+\vec{P}^{(2)}+\vec{P}^{(3)}+\vec{P}^{\prime}
$$

A first-order susceptibility tensor $\chi_{i j}^{(1)}=\left(\varepsilon_{i j}-1\right) / 4 \pi$ have two independent nonzero components: $\chi_{\perp}^{(1)}=\chi_{x x}^{(1)}=\chi_{y y}^{(1)}$ and $\chi_{\|}^{(1)}$ $=\chi_{z z}^{(1)}$. The components of the second and third-order nonresonance nonlinear polarizations have the following forms:

$$
\begin{aligned}
P_{j}^{(2)}= & \int \chi_{2, j}\left(\eta_{1}, \eta_{2}, t_{1}, t_{2} ; \alpha\right) E_{z}\left(\eta-\eta_{1}, t-t_{1}\right) \\
& \times E_{z}\left(\eta-\eta_{1}-\eta_{2}, t-t_{1}-t_{2}\right) d t_{1} d t_{2} d \eta_{1} d \eta_{2},
\end{aligned}
$$

$$
\begin{aligned}
P_{j}^{(3)}= & \int \rho_{3, j}\left(\eta_{1}, \eta_{2}, \eta_{3}, t_{1}, t_{2}, t_{3} ; \alpha\right) E_{z}\left(\eta-\eta_{1}, t-t_{1}\right) E_{z}\left(\eta-\eta_{1}\right. \\
& \left.-\eta_{2}, t-t_{1}-t_{2}\right) E_{z}\left(\eta-\eta_{1}-\eta_{2}-\eta_{3}, t-t_{1}-t_{2}-t_{3}\right) \\
& \times d \eta_{1} d \eta_{2} d \eta_{3} d t_{1} d t_{2} d t_{3}
\end{aligned}
$$

where 


$$
\begin{gathered}
\chi_{2, j}\left(\eta_{1}, \eta_{2}, t_{1}, t_{2} ; \alpha\right)=\chi_{j m n}\left(\eta_{1}, \eta_{2}, t_{1}, t_{2}\right) \frac{e_{E, m} e_{E, n}}{e_{E, z}^{2}}, \\
\rho_{3, j}\left(\eta_{1}, \eta_{2}, \eta_{3}, t_{1}, t_{2}, t_{3} ; \alpha\right) \\
=\rho_{j m n r}\left(\eta_{1}, \eta_{2}, \eta_{3}, t_{1}, t_{2}, t_{3}\right) \frac{e_{E, m} e_{E, n} e_{E, r}}{e_{E, z}^{3}}, \quad j, n, m, r=y, z,
\end{gathered}
$$

where $\chi_{j m n}$ and $\rho_{j m n r}$ are the components of tensors of the quadratic and cubic susceptibilities [4-6]. $e_{n, m}=\vec{e}_{n} \cdot \vec{e}_{m}, \vec{e}_{m}$ are unit vectors which are points along vector $\vec{E}$ and $x, y, z$ coordinate axes; $n, m=E, x, y, z, \vec{E}=\vec{e}_{E} E$, and $E_{z}=e_{E, z} E$. The unity vector $\vec{e}_{E}$ determine the direction of polarization of linear polarized optical wave (Fig. 1). For the most of noncentrosymmetric crystals $d_{i j k} \gg \rho_{i j k l}$ and usually third-order nonlinearity can be neglected. Although for the convenience in the equations we are keeping the both members $\chi_{j m n}$ and $\rho_{\text {jmnr }}$ really only one of them is not zero in dependence from this we consider noncentrosymmetric or Kerr medium.

The quantity $\vec{P}^{\prime}$ is the resonant nonlinear polarization due to the interaction of pulse with optical active impurity atoms under condition of two-photon processes. We shall assume, as is true of a large class of laser crystals (see, for example, Ref. [5]), that the vector of electric dipole moment $\vec{d}_{0}$ of impurity atoms and the optical axis $O$ of uniaxial matrix coincide. In such system the theory of self-induced transparency constructed in the work [21]. Another situation of selfinduced transparency in anisotropic media when the vector $\vec{d}_{0}$ do not coincide with the optical axis of the crystal considered in the work [22]. In the present work we assume that the vector $\vec{d}_{0}$ and the optical axis $O$ of matrix coincide and directed to the axis $z$. In such a case the vector $\vec{E}$ and the vector of polarization of the impurity atoms $\vec{P}^{\prime}$ are coupling to each other through their $z$-components $E_{z}$ and $P_{z}^{\prime}$ [21]. Consequently, we have to consider the nonlinear wave equation (1) for the $z$-component of the quantity $\vec{E}(\eta, t)$.

We can materially simplify Eq. (1), using the method of slowly changing profile. For this purpose, we represent the function $E_{z}$ and $P_{z}^{\prime}$ in the form

$$
E_{z}=\sum_{l= \pm 1} \hat{E}_{l} Z_{l}, \quad P_{z}^{\prime}=n_{0} \sum_{l} Z_{l} d_{l} \hat{E}_{-l}
$$

where $Z_{l}=\exp [i l(k \eta-\omega t)], \hat{E}_{l}$ and $d_{l}$ are the slowly changing complex amplitudes of the optical wave, and $l$ runs through the values $\pm 1, \pm 2 \ldots$. To guarantee the reality of the quantity $\hat{E}_{l}$ we set $\hat{E}_{l}=\hat{E}_{-l}^{*}$ [15]. The dependence of the quantity $d_{l}$ on the strength of the electrical field $\hat{E}_{z}$ is governed by the optical Bloch equations which are based on the representation of the resonance impurity atoms by an ensemble of two-level atoms whose evolution is caused by processes of interaction with optical extraordinary waves [11].

Substituting Eqs. (2)-(4) in Eq. (1) we obtain for the envelopes the following nonlinear wave equation

$$
\begin{gathered}
\sum_{l} Z_{l}\left\{\left[W_{l}(\alpha) \hat{E}_{l}-i \alpha_{l}(\alpha) \frac{\partial \hat{E}_{l}}{\partial \eta}+i \beta_{l}(\alpha) \frac{\partial \hat{E}_{l}}{\partial t}-\mu_{l}(\alpha) \frac{\partial^{2} \hat{E}_{l}}{\partial \eta^{2}}\right.\right. \\
\left.-\gamma_{l}(\alpha) \frac{\partial^{2} \hat{E}_{l}}{\partial \eta \partial t}-\delta_{l}(\alpha) \frac{\partial^{2} \hat{E}_{l}}{\partial t^{2}}\right]-\sum_{l^{\prime}} \chi_{l, l^{\prime}}^{(2)}(\alpha) \hat{E}_{l-l^{\prime}} \hat{E}_{l^{\prime}} \\
\left.-\sum_{l^{\prime}, l^{\prime \prime}} \rho_{l, l^{\prime}, l^{\prime \prime}}^{(3)}(\alpha) \hat{E}_{l-l^{\prime}-l^{\prime \prime}} \hat{E}_{l^{\prime}} \hat{E}_{l^{\prime \prime}}-r_{l}(\alpha)\left(\delta_{l, 1}+\delta_{l,-1}\right)\right\}=0
\end{gathered}
$$

and connect with them the system of Bloch equations for two-photon processes

$$
\begin{gathered}
\frac{\partial d_{l}}{\partial t}=i l\left(2 \omega-\omega_{21}+G \hat{E}_{l} \hat{E}_{-l}\right) d_{l}-i l \kappa_{0} N \hat{E}_{l}^{2}, \\
\frac{\partial N}{\partial t}=\frac{i}{2} \kappa_{0} \sum_{l= \pm 1} l d_{-l} \hat{E}_{l}^{2},
\end{gathered}
$$

where

$$
\begin{aligned}
& W_{l}=l^{2}\left(c^{2} k^{2} \kappa_{l}^{(2)}-\omega^{2} \kappa_{l}^{(1)}\right), \\
& \alpha_{l}=l\left(c^{2} l k^{2} A_{l}^{(2)}+2 k c^{2} \kappa_{l}^{(2)}-l \omega^{2} A_{l}^{(1)}\right), \\
& \beta_{l}=l\left(c^{2} l k^{2} B_{l}^{(2)}-2 \omega \kappa_{l}^{(1)}-l \omega^{2} B_{l}^{(1)}\right), \\
& \gamma_{l}=l\left(2 \omega A_{l}^{(1)}+l \omega^{2} T_{l}^{(1)}-2 c^{2} k B_{l}^{(2)}-c^{2} l k^{2} T_{l}^{(2)}\right), \\
& \delta_{l}=c^{2} l^{2} k^{2} D_{l}^{(2)}-l^{2} \omega^{2} D_{l}^{(1)}-2 l \omega B_{l}^{(1)}-\kappa_{l}^{(1)}, \\
& \mu_{l}=c^{2} l^{2} k^{2} C_{l}^{(2)}+c^{2} \kappa_{l}^{(2)}+2 c^{2} l k A_{l}^{(2)}-l^{2} \omega^{2} C_{l}^{(1)}, \\
& A_{l}^{(j)}=\frac{\partial \kappa_{l}^{(j)}}{\partial(l k)}, \quad B_{l}^{(j)}=\frac{\partial \kappa_{l}^{(j)}}{\partial(l \omega)}, \quad C_{l}^{(j)}=\frac{1}{2} \frac{\partial^{2} \kappa_{l}^{(j)}}{\partial(l k)^{2}}, \\
& D_{l}^{(j)}=\frac{1}{2} \frac{\partial^{2} \kappa_{l}^{(j)}}{\partial(l \omega)^{2}} \\
& T_{l}^{(j)}=\frac{\partial^{2} \kappa_{l}^{(j)}}{\partial(l k) \partial(l \omega)}, \quad j=1,2, \quad r_{l}(\alpha)=2 \pi \omega^{2} n_{0} \psi_{l}(\alpha) d_{-l}, \\
& \psi_{l}(\alpha)=1-\frac{k^{2} c^{2}}{\omega^{2}} \cos ^{2} \alpha \varepsilon_{\perp}^{-1}(l \omega, l k), \quad \kappa_{l}^{(1)}=\varepsilon_{\|}(l k, l \omega), \\
& \kappa_{l}^{(2)}=\int\left\{\delta(\eta) \delta(t)+4 \pi \cos ^{2} \alpha\right. \\
& \left.\times\left[\chi_{\|}^{(1)}(\eta, t)+\tan \alpha \chi_{\perp}^{(1)}(\eta, t) \frac{e_{E, y}}{e_{E, z}}\right]\right\} e^{i l(\omega t-k \eta)} d t d \eta,
\end{aligned}
$$




$$
G=\frac{r_{22}-r_{11}}{4 \hbar}, \quad \kappa_{0}=\frac{\left|r_{21}\right|^{2}}{2 \hbar}, \quad r_{i i}=\frac{2}{\hbar} \sum_{n>3} \frac{\left|\mu_{i m}\right|^{2} \omega_{m i}}{\omega_{m i}^{2}-\omega^{2}}, \quad r_{21}=r_{12}^{*}=\sum_{n>3} \frac{\mu_{1 m} \mu_{m 2}}{\hbar\left(\omega_{m 2}+\omega\right)}, \quad i=1,2 .
$$

Here $\hbar$ is Planck's constant, $\omega_{n m}$ and $\mu_{n m}$ are the frequencies and matrix elements of electric-dipole moments transitions between $n$ and $m$ level of energy of the two-level impurity optical active atoms. The effective susceptibilities of the second and third order in uniaxial media for the quantity $E_{z}$ have the following form:

$$
\begin{aligned}
\chi_{l, l^{\prime}}^{(2)}(\alpha)= & 4 \pi l^{2} \omega^{2}\left[\psi_{l}(\alpha)\left(\chi_{z y y} \lambda_{l}^{2} \cot ^{2} \alpha+\chi_{z z z}\right)+\lambda_{l} \cos ^{2} \alpha\right. \\
& \left.\times\left(\chi_{y y z}+\chi_{y z y}\right)-\chi_{y y y} \cot \alpha \cos ^{2} \alpha \lambda_{l}^{2}\right], \\
\rho_{l, l^{\prime}, l^{\prime \prime}}^{(3)}(\alpha)= & 4 \pi l^{2} \omega^{2}\left[\psi_{l}(\alpha) \lambda_{l}^{2} \cot ^{2} \alpha\left(\rho_{z z y y}+\rho_{z y y z}+\rho_{z y z y}\right)\right. \\
& +\psi_{l}(\alpha) \rho_{z z z z}-\psi_{l}(\alpha) \lambda_{l}^{3} \rho_{z y y y} \cot ^{3} \alpha+\lambda_{l} \cos ^{2} \alpha \\
& \times\left(\rho_{y y z z}+\rho_{y z y z}+\rho_{y z z y}\right)+\cot ^{2} \alpha \cos ^{2} \alpha \lambda_{l}^{3} \rho_{y y y y} \\
& \left.-\cos ^{2} \alpha \cot \alpha\left(\rho_{y y y z}+\rho_{y y z y}+\rho_{y z y y}\right) \lambda_{l}^{2}\right],
\end{aligned}
$$

where

$$
\begin{aligned}
\lambda_{l} & =\frac{\varepsilon_{\|}(l k, l \omega)}{\varepsilon_{\perp}(l k, l \omega)}, \quad \chi_{i j n}=\chi_{i j n}\left(l k, l \omega, l^{\prime} k, l^{\prime} \omega\right), \\
\rho_{i j n m} & =\rho_{i j n m}\left(l k, l \omega, l^{\prime} k, l^{\prime} \omega, l^{\prime \prime} k, l^{\prime \prime} \omega\right), \quad i, j, n, m=y, z .
\end{aligned}
$$

\section{OPTICAL TWO-PHOTON BREATHER}

The further analysis of the set of equations (5) and (6), we make use of the perturbative reduction method, developed in Refs. [23,24]. Under the condition $\left|\Theta_{l}\right| \ll 1$, the quantities $\hat{E}_{l}$ can be represented as

$$
\hat{E}_{l}(\eta, t)=\sum_{\alpha=1}^{\infty} \sum_{n=-\infty}^{+\infty} \varepsilon^{(\alpha)} Y_{n} \varphi_{l, n}^{(\alpha)}(\zeta, \tau)
$$

where $\Theta_{l}(\eta, t)=\kappa_{0} \int_{-\infty}^{t} \hat{E}_{l}^{2}\left(\eta, t^{\prime}\right) d t^{\prime}$ is the quantity proportional to the energy of the optical pulse in the case of twophoton transition, $Y_{n}=e^{i n(Q \eta-\Omega t)}, \zeta=\varepsilon Q(\eta-v t), \tau=\varepsilon^{2} t, v$ $=d \Omega / d Q$, and $\varepsilon$ is the small parameter.

Such a representation allows us to separate from $\hat{E}_{l}$ the still more slowly changing quantity $\varphi_{l, n}^{(\alpha)}$. Consequently, it is assumed that the inequalities $\omega \gg \Omega, k \gg Q,\left|\partial \varphi_{l, n}^{(\alpha)} / \partial t\right|$ $\ll \Omega\left|\varphi_{l, n}^{(\alpha)}\right|,\left|\partial \varphi_{l, n}^{(\alpha)} / \partial \eta\right| \ll Q\left|\varphi_{l, n}^{(\alpha)}\right|$ are satisfied. In the interaction of an optical pulse with a resonantly absorbing medium, the most significant effects are usually observed at exact resonance.Therefore, for simplicity, we consider the system of Bloch equations (6) at exact resonance, i.e., with $2 \omega$ $=\omega_{21}$.

For the determination of the explicit form of the quantity $P_{z}^{\prime} \quad$ we expand the quantities $d_{l}=\Sigma_{\alpha=1} \varepsilon^{\alpha} b_{l}^{(\alpha)}$ and $N$ $=\sum_{\alpha=0} \varepsilon^{\alpha} N^{(\alpha)}$ in a perturbation-theory series in the small non- linear parameter $\varepsilon$. Substituting these expansions and expression (9) in the set of equations (6) we obtain

$$
P_{z}^{\prime}=\varepsilon^{3} \sum_{l, n, n^{\prime}, m} Z_{l} Y_{n} \frac{l R_{l, 0}(\alpha)}{n-n^{\prime}} \varphi_{l, n-n^{\prime}-m}^{(1)} \varphi_{l, m}^{(1)} \varphi_{-l, n^{\prime}}^{(1)}
$$

where

$$
R_{l, 0}(\alpha)=\frac{4 \pi n_{0} \kappa_{0} \omega^{2} \tau_{0}}{\hbar \Omega} \psi_{l}(\alpha) \quad\left(\tau_{0}= \pm 1\right)
$$

is the optical resonance absorption coefficient under the condition of TPEI $[9,11]$. The positive sign of the quantitiy $\tau_{0}$ corresponds to the attenuating medium, the negative sign corresponds to the amplifier medium.

From Eq.(10) it is clear that, unlike one-photon processes [15], the resonance nonlinear polarization $P_{z}^{\prime}$ does not contain a linear part related to $\hat{E}_{l}$, but consists only of a nonlinear part.

Substituting Eqs. (9) and (10) into Eq.(5) we obtain the nonlinear Schrödinger equation (NSE) for the quantities $\psi_{l,-l}=\varepsilon \sqrt{q_{l}} \varphi_{l,-l}^{(1)}($ for $l= \pm 1)$ :

$$
i l \frac{\partial \psi_{l,-l}}{\partial t}+\frac{\partial^{2} \psi_{l,-l}}{\partial y_{l}^{2}}+\left|\psi_{l,-l}\right|^{2} \psi_{l,-l}=0
$$

where

$$
y_{l}=\frac{\eta-v_{g} t}{\sqrt{p_{l}}}, \quad p_{l}=-\frac{1}{2} \frac{\partial^{2} \Omega}{\partial Q^{2}}, \quad q_{l}=\frac{m_{l}-R_{l, 0}}{2 \Omega \delta_{l}-l \beta_{l}-Q \gamma_{l}} .
$$

The nonresonance nonlinear term

$$
m_{l}=M_{l}=\frac{16 \pi^{2} \omega^{4}\left(\chi_{l,-l}^{(2)}+\chi_{l, 2 l}^{(2)}\right) \chi_{2 l, l}^{(2)}}{W_{2 l}-2 l \alpha_{l} Q-2 l \beta_{l} \Omega-4 \gamma_{l} Q \Omega+4 \delta_{l} \Omega^{2}+4 \mu_{l} Q^{2}}
$$

for quadratic nonlinearity crystals and

$$
m_{l}=L_{l}=4 \pi \omega^{2}\left(\rho_{l, l,-l}^{(3)}+\rho_{l,-l, l}^{(3)}\right)
$$

for crystals with cubic nonlinearity.

Substituting the soliton solution of the NSE for superenvelope $\psi_{l,-l}$, into Eq.(9), we obtain the following form for the envelope $\hat{E}_{l}$ of the breather solution from the wave equation (5) under the condition $p_{l} q_{l}>0[1-3,9,15,24]$ :

$$
\hat{E}_{l}=\frac{2 i l \eta_{0}}{\sqrt{q_{l}}} \frac{e^{-i l\left(\varphi_{1, l}+\Omega t-Q \eta\right)}}{\cosh 2 \eta_{0} \varphi_{2, l}},
$$

where

$$
\varphi_{1, l}=\frac{2 \xi_{0} \eta}{\sqrt{p_{l}}}+2\left[2\left(\xi_{0}^{2}-\eta_{0}^{2}\right)-\frac{\xi_{0} v}{\sqrt{p_{l}}}\right] t-\varphi_{0}
$$



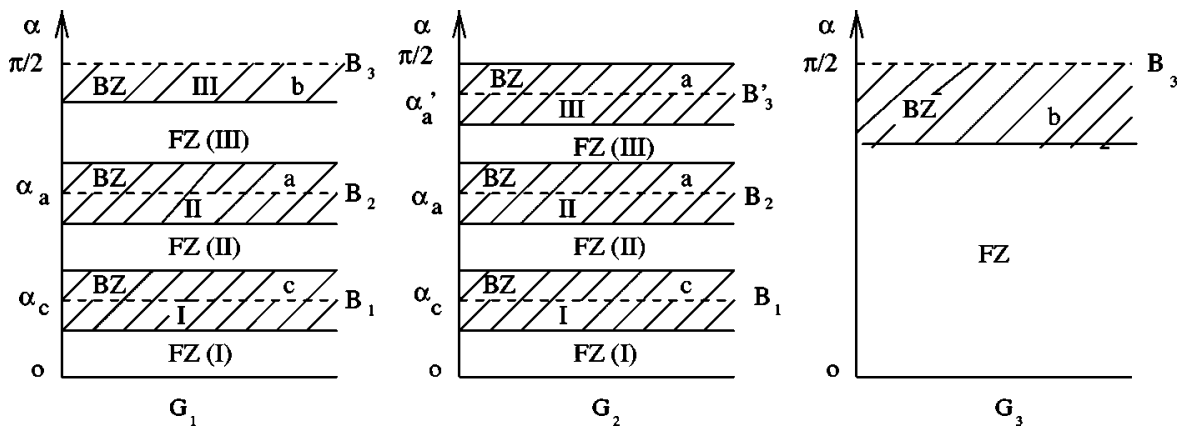

FIG. 2. There are three different SBZ realizations for the $G_{1}, G_{2}$, and $G_{3}$ of crystals with quadratic nonlinearity. For crystals with cubic nonlinearity the SBZ coincides with the SBZ of $G_{2}$ of the quadratic nonlinearity crystals. The optical breathers of the small energy are formed in the zones I, II, and III, (BZ) by means of three different $(a),(b)$, and $(c)$ mechanisms as shown in the figures. These zones are hatched. The width of the zones depends on the nonlinearity parameters and from this one- or two-photon processes are realized.

$$
\varphi_{2, l}=\frac{\eta}{\sqrt{p_{l}}}+\left(4 \xi_{0}-\frac{v}{\sqrt{p_{l}}}\right) t-y_{0} .
$$

The quantities $\xi_{0}, \eta_{0}, \varphi_{0}$ and $y_{0}$ denote the scattering date of the inverse scattering transform $[1,24]$ when applied to the nonlinear equation.

The appearance of the factor $e^{i l(Q \eta-\Omega t)}$ in the breather expression (13) indicates the formation of periodic, and slow in comparison with $Z_{l}$, beats in coordinates and time, with characteristic parameters $\Omega \ll \omega$ and $Q \ll k$. The equation $W_{l= \pm 1}$ $=0$ provides the dispersion law for extraordinary waves and the relation between $\Omega$ and $Q$, for fixed values of $l$ and $n$ equal to \pm 1 , has the form

$$
\alpha_{l} n Q+\beta_{l} n \Omega-\gamma_{l} Q \Omega+\mu_{l} Q^{2}+\delta_{l} \Omega^{2}=0 .
$$

Because all coefficients of this equation are functions of $\alpha$ the connection between quantities $\Omega$ and $Q$ will depend on the angle $\alpha$ too.

\section{STRUCTURE OF BREATHERS ZONE}

The quantity $q_{l}$ contains terms coming both from the resonance $R_{l, 0}$ and nonresonance $m_{l}$ nonlinear terms. Depending on the values of these quantities, different mechanisms of the formation of optical breathers can take place.

(a) $m_{l}=R_{l, 0}$ and $m_{l} R_{l, 0}<0$. This is the condition of the realization of the "blended" MFB when both the nonresonance and resonance nonlinearities are simultaneously effective and act together with the dispersion in the process of the formation of resonance optical breathers of the small energy.

(b) $m_{l} \ll R_{l, 0}$. The pulse interaction with optical impurities has nonlinear character and nonresonance interactions are ignored. This situation corresponds to the two-photon self-induced transparency and resonance optical breathers of the small energy $[2,3]$.

(c) $m_{l} \gg R_{l, 0}$ The pulse interaction with optical impurities does not contribute to the formation of the nonresonance breathers [9].

From the expressions (7), (8), and (11) it is clear that the quantities $m_{l}$ and $R_{l, 0}$ depend not only differently on the direction of wave propagation, but also essentially on the symmetry of the medium. Hence the mechanisms of the forma- tion of the optical breathers of extraordinary waves, which are determined by means of the quantities $m_{l}$ and $R_{l, 0}$ will depend both on the direction of propagation of the pulses and on the symmetry of the medium. Thus we expect that several chosen wave propagation directions $B$ exist in uniaxial crystals at which different mechanisms [(a), (b), and (c)] of the formation of the optical breathers are effectively contributing. In Fig. 1 direction of the wave propagation coincide with one of these $B$ chosen directions. In Figs. 1-3, these directions are shown by dashed lines. Breather zones (BZ) correspond to the hatched regions around these directions where one of the MFB will be most effective. There are forbidden zones (FZ) located between the BZ where the MFB are not or only weakly effective (Figs. 2 and 3).

In order to find these directions we have to analyze the symmetry of the media. Analyzing the expression (7), we can separate all quadratic uniaxial crystals into the three groups: The first group $\left(G_{1}\right)$ contains the crystals with the crystal classes (CC) $\overline{4}, \overline{6}$ and $\overline{6} m 2$ of the uniaxial tetragonal and hexagonal crystal systems; the second group $\left(G_{2}\right)$ contains the crystals with the $\mathrm{CC} 3,3 \mathrm{~m}, 4,4 \mathrm{~mm}, 6,6 \mathrm{~mm}$ of the trigonal, tetragonal, and hexagonal crystal systems; and the third group $\left(G_{3}\right)$ contains the crystals with the CC 32, 422,

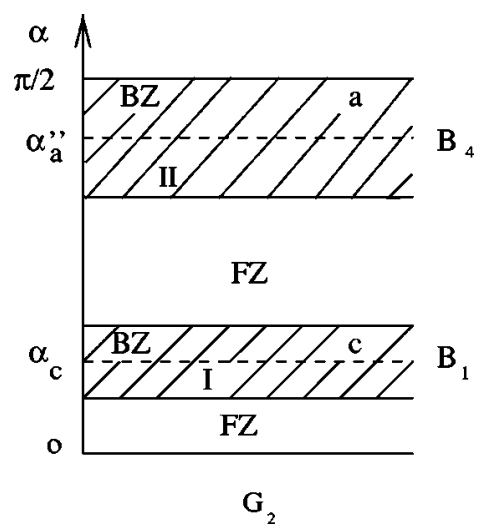

FIG. 3. There is the SBZ for the quadratic $G_{2}$ and cubic crystals, under the condition of two-photon processes, when adjacent BZ II and BZ III somewhat overlap with one another (in Fig. $2 G_{2}$ ) and combine into a one wide BZ II which by means of the directions $B_{4}$ and angle $\alpha_{a}^{\prime \prime}$ are determined. 
$\overline{42} m$ and 622 of the trigonal, tetragonal and hexagonal crystal systems.

The situation considered in Fig. $2 G_{1}$ taking into account that for the group of crystals $G_{1}$ the quantity $\chi_{l, l^{\prime}}^{(2)}(\alpha \rightarrow \pi / 2)$ $=0$. Unlike the $G_{1}$, the $G_{2}$ of the crystals realizes another SBZ (see Fig. $2 G_{2}$ ). In particular, for the $G_{2}$ the quantity $\chi_{l, l^{\prime}}^{(2)}(\alpha \rightarrow \pi / 2) \neq 0$ and consequently in the zone III the direction $B_{3}^{\prime}$ the angle $\alpha_{a}^{\prime}$ appear which are defined through the equation $M_{l}=R_{l, 0}$ (but for a different value $\chi_{l, l^{\prime}}^{(2)} \sim \chi_{z z z}$ than in zone II). In zones II and III resonance optical breathers will be formed by means of the mechanism (a) but breather parameters in zones II and III will be different.

For the $G_{3}$ of the crystals the situation is quite different as compared to $G_{1}$ and $G_{2}$. In this case all components of the quantity $\chi_{l, l^{\prime}}^{(2)}$ equal to zero and consequently $M_{l}=0$ everywhere and we have only one special direction $B_{3}$ which points along the $y$ axis. The quantity $R_{l, 0}(\pi / 2)$ has maximum value in this direction meaning that in this single BZ the (b) MFB (i.e., self-induced transparency) will be realized (see Fig. $2 G_{3}$ ).

The SBZ considered above in Fig. 2 are valid for OPEI as well as for TPEI. These cases differ from each other only by the value of $R_{l, 0}$, which for the case of the TPEI is rather small in comparison with the case of OPEI [15]. Consequently, the equation $m_{l}=R_{l, 0}$ necessary for realization of the "blended" MFB under the condition of the TPEI will be satisfied for the directions of the pulse for which the angle $\alpha$ will be closer to $\pi / 2$ (at the same value of all other parameters of pulse and medium). This is due to $R_{l, 0}$ taking maximum value when the pulse along the $y$ axis is propagated. This fact lead to the following differences between the SBZ for the one-and two-photon breathers (OPEI and TPEI processes).

\section{A. In the quadratic nonlinearity crystals}

(1) For the two-photon breathers comparison with onephoton breathers the FZ (II) and FZ(III) for the crystals $G_{1}$ and $G_{2}$ and also FZ for the crystals $G_{3}$ will be extended. At the same time BZ II and BZ III for crystals $G_{1}$ and $G_{2}$, and BZ for the crystals $G_{3}$ will be narrowed (Fig. 2).

(2) In the case of the two-photon breathers for the crystals $G_{2}$ will be able to arise different, comparison with Fig. 2 $G_{2}$, SBZ when width of the FZ (III) equal to zero. In this case adjacent zones of BZ II and BZ III somewhat overlap with one another and combine into a one wide BZ with chosen direction $B_{4}$ makes angle $\alpha_{a}^{\prime \prime}$ with the optical $O$ axis, which do not coincide in ether directions $B_{2}$ or $B_{3}^{\prime}$ directions. In other words, under the condition of TPEI for the crystals $G_{2}$ a new SBZ can arise (Fig. 3).

Consequently, unlike the OPEI, under the condition of the TPEI processes for the crystals $G_{2}$, depending on the value of the parameters of the pulses and media, two different Fig. $2 G_{2}$ and Fig. 3 SBZ will be realized.

(3) The SBZ for crystals with quadratic susceptibility $G_{1}$ and $G_{3}$ are the same for one- and two-photon breathers.

\section{B. In Kerr-media}

From Eq. (8) it follows that all uniaxial crystals with cubic nonlinearity have the same chosen directions and SBZ as those of the crystals of $G_{2}$ with quadratic nonlinearity and hence Fig. $2 G_{2}$ and Fig. 3 applies for crystals with cubic nonlinearity as well.

Hence for crystals with quadratic susceptibility $G_{2}$ and Kerr media SBZ will be different on whether the conditions of OPEI or TPEI are realized, at the same values of the other parameters of the medium and field.

\section{CONCLUSION}

The NSE is the fundamental equation to describe solitary waves, which occur when dispersion is balanced by nonlinearity, when both group velocity dispersion and nonlinearity play an important role simultaneously. The input intensity $I_{s o l}$ of the pulse necessary for the excitation of the soliton by means of the quantity $\partial^{2} k / \partial \omega^{2}$, i.e., from the dispersion properties $k(\omega)$ of the medium is determined [8]. For the breathers of small energy the situation is different. An input intensity for the breather generation $I_{b r}$ is proportional to the quantity $\partial^{2} \Omega / \partial Q^{2}$ and it can be determined from the relation (14) for $\Omega$ and $Q$. The quantities $\Omega$ and $Q$ characterize "internal properties" of the breather. Unlike soliton, the intensity of excitation of the breather $I_{b r}$ is determined not only by the dispersive properties of the medium and the direction of extraordinary wave propagation, but also by the internal parameters of the breather and the symmetry of the medium. Because numerical values of the nonlinear susceptibilities can vary very strongly in different media, in different solids the minimum intensity for non-resonance breather generation will be different, too [15].

Unlike one-photon breathers [15], for two-photon breathers connection between breather's parameters $\Omega$ and $Q$ [Eq. (14)] and the quantity $M_{l}$ [Eq. (12)] do not depend on the coefficient of resonance optical absorption $R_{l, 0}(\alpha)$ [Eq. (11)]. Therefore, unlike of the case of OPEI, under the condition of TPEI the pulse interaction with optical resonance impurities does not influence the parameters of the nonresonance breathers (BZ I in Fig. $2 G_{1}$ and Fig. $2 G_{2}$ and Fig. 3). We have to note that under the condition of the OPEI linear part of the atom-photon interaction influence on the parameters of the nonresonance breathers $[9,15]$.

Consequently in the anisotropic uniaxial media three mechanisms [(a), (b), and (c)] of the formation of the optical breathers can be realized for different directions of the extraordinary wave propagation depending on the symmetry of the medium and from this one- or two-photon processes is realized. The uniaxial crystals with quadratic nonlinearity can be divided into three different groups with each of them having its own SBZ. The SBZ within one of these groups does not depend on the crystal systems (syngonies or point groups) and is determined by means of CC and are different for one- or two-photon processes.

Unlike quadratic media for uniaxial crystals with cubic nonlinearity, the SBZ depends neither on the crystal systems nor on the CC and only depends on which one- or twophoton processes are realized. The SBZ for cubic crystals coincides with the SBZ of the $G_{2}$ of the crystals with quadratic nonlinearity.

Hence the mechanisms of the formation of breathers depend on the direction of the pulse propagation and from this 
one- or two-photon processes are realized and this dependence is qualitatively different for media with quadratic and cubic susceptibilities.

Comparison our results with Ref. [15] for OPEI, have shown that the SBZ depends not only from CC but from this one- or two-photon processes are realized.

The mechanisms (b) and (c) do not act independently but also influence each other. They can strengthen each other under the condition of (a) MFB but in another case when $m_{l}-R_{l, 0}=0$, for example in amplifier media at $\tau_{0}<0$, the breathers do not exist and consequently the SBZ will be changed significiantly: BZ II in Fig. $2 G_{1}$, BZ II and BZ III in Fig. $2 G_{2}$ and BZ II in Fig. 3 will be transformed to the FZ where no breathers exist.
These considered mechanisms can be realized in different isotropic media too. Among them one of the most interesting for applications could be doped fiber media: e.g., an erbiumdoped optical fiber in which a "blended" mechanism for the formation of solitons has already been considered $[18,19]$. We anticipate that the properties of optical small energy breathers may become very useful to the future of optical communications and photonic devices.

\section{ACKNOWLEDGMENTS}

G.T.A. would like to thank the Max Planck Institute for the Physics of Complex Systems and the University of Central Florida for their hospitality. This research has been supported in part by NSF Grant No. 0129714.
[1] A. C. Newell, Solitons in Mathematics and Physics (SIAM, Philadelphia, PA, 1985).

[2] G. T. Adamashvili and S. V. Manakov, Solid State Commun. 48, 381 (1983); G. T. Adamashvili, Opt. Spektrosk. 176, 1400 (1992); 76, 3 (1994); 80, 251 (1996).

[3] D. J. Kaup, Phys. Rev. A 16, 704 (1977); D. J. Kaup and L. Scacca, J. Opt. Soc. Am. 70, 224 (1980).

[4] A. Yariv and P. Yeh, Optical Waves in Crystals (Wiley, New York, 1984); A. Yariv, Quantum Electronics (Wiley, New York, 1989).

[5] A. A. Kaminskii, Lasernie Kristali (Nauka, Moscow, 1975).

[6] Nelineinaia Spectroskopia, edited by N. Bloembergen (Mir, Moscow, 1979) (in Russian).

[7] A. P. Suchorukov, Nelineinie Volnovie Vzaimodeistvia v Optike i Padiophizike (Nauka, Moscow, 1988), p 232.

[8] E. G. Sauter, Nonlinear Optics (Wiley, New York, 1996).

[9] G. T. Adamashvili and A. A. Maradudin, Phys. Rev. E 55, 7712 (1997).

[10] S. L. McCall and E. L. Hahn, Phys. Rev. 183, 457 (1969).

[11] L. Allen and J. N. Eberly, Optical Resonance and Two Level Atoms (Wiley-Interscience, New York, 1975); A. I. Maimistov, A. M. Bahsarov, S. O. Elyutin, and Yu. M. Sklyarov, Phys. Rep. 191, 1 (1990).

[12] G. T. Adamashvili, Phys. Lett. A 208, 323 (1995); Opt. Spektrosk. 85, 95 (1998).
[13] J. C. Diels and E. L. Hahn, Phys. Rev. A 10, 2501 (1974).

[14] J. E. Rothenberg, D. Grishkovsky, and A. C. Balant, Phys. Rev. Lett. 53, 552 (1984).

[15] G. T. Adamashvili, Phys. Rev. E 69, 026608 (2004).

[16] G. T. Adamashvili, Phys. Lett. A 138, 304 (1989); Zh. Eksp. Teor. Fiz. 97, 234 (1990); Teor. Mat. Fiz. 99, 92 (1994).

[17] A. I. Maimistov and E. A. Manykin, Zh. Eksp. Teor. Fiz. 85, 1177 (1983).

[18] M. Nakazawa, E. Yamada, and H. Kubota, Phys. Rev. Lett. 66, 2625 (1991); Phys. Rev. A 44, 5973 (1991); E. J. S. Fonseca, S. B. Cavalcanti, and J. M. Hickmann, Phys. Rev. E 64, 016610 (2001).

[19] D. P. Caetano, S. B. Cavalcanti, J. M. Hickmann, A. M. Kamchatnov, R. A. Kraenkel, and E. A. Makarova, Phys. Rev. E 67, 046615 (2003).

[20] L. D. Landau and E. M. Lifshitz, Electrodynamics of Continuous Media (Pergamon, New York, 1960).

[21] V. M. Agranovich, G. T. Adamashvili, and V. I. Rupasov, Zh. Eksp. Teor. Fiz. 80, 1741 (1981).

[22] G. T. Adamashvili and M. D. Peikrishvili, Opt. Spektrosk. 89, 314 (2000).

[23] T. Taniuti and N. Iajima, J. Math. Phys. 14, 1389 (1973).

[24] R. K. Dodd, J. C. Eilbeck, J. D. Gibbon, and H. C. Morris, Solitons and Nonlinear Wave Equation (Academic, New York, 1982). 